\title{
Selected High and Low Pollen Hoarding Colonies of Apis mellifera for Apple Foraging Preference
}

\author{
Harish Kumar Sharma*, Monika, Kiran Rana and Meena Thakur \\ YSP University of Horticulture and Forestry, Department of Entomology, \\ Nauni, Solan, Himachal Pradesh-173230, India \\ *Corresponding author
}

\begin{tabular}{|c|c|}
\hline & A B S T R A C T \\
\hline & \multirow{8}{*}{$\begin{array}{l}\text { The data on performance of high and low pollen hoarding colonies revealed significant } \\
\text { differences with respect to different colony parameters. Ranking obtained by different } \\
\text { colonies with respect to colony strength, brood area, pollen stores, honey stores, pollen } \\
\text { stores and hygienic behaviour were scored as per their performance. The main criteria for } \\
\text { selecting colonies with high pollen hoarding and low pollen hoarding tendency was } \\
\text { average pollen stores. The three high pollen hoarding colonies (H- } 4 \text {, H-5 and H-9) were } \\
\text { those which scored } 3 \text { to } 4.50 \text {, whereas low pollen hoarding colonies (L-4, L- } 6 \text { and L9) } \\
\text { scored } 4 \text { to } 4.50 \text { for average pollen stores and bee foragers. The low pollen hoarding \& } \\
\text { high pollen hoarding colonies also showed significant seasonal variations with respect to } \\
\text { various colonies characteristics. Number of incoming bees with pollen loads at hive } \\
\text { entrance was significantly more ( } 51.94 \text { bees per minute) in high pollen hoarding honeybee } \\
\text { colonies as compared to low pollen hoarding colonies ( } 42.82 \text { bees per minute). Apple } \\
\text { pollen preference in colony number G- } 2 \text { from general stock showed highest preference for } \\
\text { apple pollen foraging with } 18.78 \text { per cent of apple pollen loads. Colony H- } 4 \text { recorded } \\
\text { minimum percentage of apple pollen load. Out of nine, } 7 \text { colonies collected significantly } \\
\text { same percentage of apple pollen. These studies suggest that selection efforts for bees with } \\
\text { the preference for apple pollen could be combined with selection for pollen hoarding to get } \\
\text { a strain of honeybee with higher preference for apple pollen foraging. }\end{array}$} \\
\hline & \\
\hline $\begin{array}{l}\text { Apis mellifera, } \\
\text { Colony strength. }\end{array}$ & \\
\hline $\begin{array}{l}\text { Colony strength, } \\
\text { Brood area, Honey }\end{array}$ & \\
\hline $\begin{array}{l}\text { stores, Hygienic } \\
\text { behaviour and } \\
\text { Pollen stores. }\end{array}$ & \\
\hline Article Info & \\
\hline $\begin{array}{l}\text { Accepted: } \\
\text { 21 September } 2017 \\
\text { Available Online: } \\
10 \text { October } 2017\end{array}$ & \\
\hline & \\
\hline
\end{tabular}

\section{Introduction}

Apple is highly cross pollinated crop grown in temperate region of the world. In India, apple cultivation is mostly confined to the states of Jammu \& Kashmir, Himachal Pradesh and Uttrakhand which together accounts for 99 per cent of the total production and the remaining is contributed by the states of Arunachal Pradesh, Nagaland, Tamilnadu and Sikkim. In Himachal Pradesh, total area under apple cultivation is 109553 ha which accounts for the production of 625199 MT fruits (Anonymous, 2015).
Globally, Apis mellifera is considered to be the most important pollinator of apple, and is managed as a commercial pollinator in all apple-growing regions of the world (Brittain 1933; Gupta et al., 1993).

Managed honey bee colonies are moved into apple orchards for pollination during the flowering period, and then removed to lower the risk of exposure to agricultural chemicals and other management practices following pollination. 
The European honey bee (Apis mellifera) is the basis of flourishing bee industry the world over. Since its introduction in India, a lot of need based research has been done on various aspects of its seasonal management, bee flora, bee behaviour. Thakur (1994) selected better honey producing breeder colonies on the basis of biological and economic characters viz. colony strength, honey stores, brood area and pollen stores. It is thus evident that there are possibilities of improvement of many characteristics of honeybee by means of selection and selecting mating of better bee stock. However, bee breeding and stock improvement remained neglected in India (Singh, 2007). Therefore, selection was greatly needed to identify honeybee colonies possessing desirable traits for further breeding programmes.

The viability of pollen carried on the body of pollen collector is higher on the body of nectar collector (Kendall, 1973) and pollen collectors have lower consistency to one cultivar of apple as compared to nectar collector (Robinson, 1979). Thus proportion of pollen collector is important for improving the effectiveness of colony for better pollination. The tendency of honey bee colonies to collect pollen has a strong genetic component (Page et al., 2000) and pollen hoarding by honey bees can be modified by selection. The present study is thus aimed at selecting high pollen hoarding honey bee colonies in the existing stock for effective pollination in apple. Keeping this in view, present study was proposed with the following objective: To select A. mellifera colonies with higher apple pollen foraging preference for effective pollination.

\section{Materials and Methods}

Data on following parameters was recorded in 20 selected colonies at 21 days interval from August, 2016 to February, 2017.
Colony strength- The strength of each experimental colony for the experiment was estimated by observing the number of frames covered with bees.

Brood area- Brood area in the experimental colonies was measured with the help of 1 inch wire grid fixed on a brood frame. The brood area was converted in to $\mathrm{cm}^{2}$ by multiplying the number of squares with a factor of 6.45 .

Pollen stores- The pollen stores were also measured in $\mathrm{cm}^{2}$ by counting the number of cells in the wire grid covered by pollens.

Honey stores- The honey stores of the colonies were measured visually on the assumption that one Langstroth frame sealed with honey weighed $2 \mathrm{~kg}$.

Bee activity - The number of bees leaving the hive and those landing at the entrance per minute from each colony were recorded in each experimental colonies. The proportion for pollen foragers was worked out in case of incoming bees. The activity was recorded 3 times a day at an interval of 21 days.

Hygienic behaviour - Hygienic behaviour was determined by pin killing 25 capped pupae. The pupae in the sealed stage were killed by piercing the caps with sharp needle. The pierced area was observed after 24 hours for removal of pin killed brood. The per cent removal of killed brood was worked out by following formula.

Number of pin killed pupae removed Per cent removal = --------------------- X 100 25

The data on hygienic behaviour was taken at 21 days interval in all experimental colonies.

Temperament - Temperament of A. mellifera colonies was determined by observing the 
behaviour of bees while handling. Working scale for temperament was kept from rank 1 to 5, rank 1 being the sign of gentle temperament and 5 being furious temperament.

Disease status - Visual observations on the incidence of brood and adult disease were recorded at 21 days interval in experimental colonies while examining colonies regularly.

Pollen stores - Data on pollen area was recorded at 21 days interval in all the experimental colonies. The pollen area at 10 days interval was also recorded to generate better information on pollen hoarding by individual colony (Hellmich et al., 1985).

\section{Selection of better performing high pollen} hoarding and lows pollen hoarding colonies

The performance of these colonies on all recorded parameters was ranked on 5 scale basis. Three high pollen hoarding and three low pollen hoarding colonies were selected on the basis of their high average performance with regard to colony strength, brood area, pollen stores, honey stores, hygienic behaviour and foraging activity at 21 days interval. The score were computed separately for low pollen hoarding and high pollen hoarding colonies. The score obtained for colony strength, brood area, pollen stores, honey stores and hygienic behavior by each colony were averaged and were considered as criteria for their general and behavioral performance. The performance of these colonies for pollen stores and incoming pollen foragers recorded at 10 days interval were averaged separately. On the basis of their performance for pollen storage and incoming pollen foragers, three best performing colonies each from low pollen hoarding stock and high pollen hoarding stock were selected. The performance of top performing colonies with respect to their general and behavioral characters was also considered. It was also ensured that selected colonies were disease free and having gentle temperament.

Three colonies were randomly selected from general stock of A. mellifera maintained in University apiary.

\section{Evaluation of selected and general stock for apple foraging preference}

In the $2^{\text {nd }}$ week of April, nine colonies were evaluated for their preference in apple flowering. These colonies were shifted to apple orchard at Regional Horticulture Research Station Mashobra when the trees were at 10 per cent bloom in apple and observations on following parameters were recorded:

\section{Colony records}

Colony strength, brood area, pollen area and honey stores of 9 selected colonies were recorded at 10 days interval as described above.

\section{Proportion of pollen collectors}

Observations on the proportions of pollen collectors in the experimental honey bee colonies were recorded by visually counting the incoming bees with or without pollen loads into a hive. To determine this number, counts of incoming bees per minute were made at the entrance of hive. The data was recorded during onset of bloom, full bloom and end of bloom at 1000 to 1200 hours for 15 sunny days during the month of April, 2017. The mean of these observations, represented the proportion of pollen foragers.

\section{Preference of $A$. mellifera for pollen foraging of apple}

Pollen detached in pollen trap from the corbiculae was collected into a plastic pollen collecting tray covered with a wire-grid to 
exclude bees. The selected experimental colonies were used for pollen trapping. Observations were recorded during onset of bloom, full bloom and end of bloom of the apple flowers at 1000 to 1200 hours for 3 sunny days. Pollen loads of incoming bees were collected with the help of pollen trap. 50 pollen pellets were randomly collected for each observation in all the colonies. These were examined under microscope and verified for presence of apple pollen grains from standard apple pollen slides. On the basis of these observations, proportion of bees with apple pollen was worked out.

\section{Statistical analysis}

The data recorded for different parameters was transformed using different transformations according to the data to be evaluated and subjected to randomized block design (RBD) (Gomez and Gomez, 1986).

\section{Results and Discussion}

The data on performance of high pollen hoarding colonies (Table 1) during August, 2016 to February, 2017 revealed that there were significant differences in different colonies with respect to colony strength. The maximum average colony strength was found in colony number H-5 (8.29 bee frames). The average pollen stores in high pollen hoarding colonies ranged from $134.36 \mathrm{~cm}^{2}(\mathrm{H}-8)$ to $628.29 \mathrm{~cm}^{2}$ (H-9) with statistically nonsignificant variations. Out of ten high pollen hoarding colonies, colony number H-5 stored average maximum amount of honey (1045 g). The average amount of pollen collected by the bees of colony number $\mathrm{H}-5$ was maximum $\left(660.43 \mathrm{~cm}^{2}\right)$.

The data on average performance of low pollen hoarding colonies (Table 2) revealed that colony number L-9 had the highest colony strength (6.29 bee frames). The average maximum amount of brood reared by the colony number L-7 was $2627 \mathrm{~cm}^{2}$. The pollen stores were maximum in colony number L-7 $\left(531.68 \mathrm{~cm}^{2}\right)$. Maximum amount of honey stores were $397.29 \mathrm{~g}$ in colony number L-4. The data on pollen stores revealed that maximum pollen stores were recorded in L-7 $\left(523.81 \mathrm{~cm}^{2}\right)$. Colony number L-6 stored significantly less pollen (238.33 $\mathrm{cm}^{2}$ ) than the rest of the colonies.

The ranking was done for the different parameters and least ranking i.e. 1 was given to least performing colony, whereas the best performing colony was ranked the highest i.e. 5. Ranking obtained by different colonies with respect to colony strength, brood area, pollen stores, honey stores, pollen stores and hygienic behaviour (Table 3) were scored as per their performance. The average score for pollen stores at 10 days interval and incoming pollen foragers ranged from 2.40 to 4.20 for high pollen hoarding colonies, whereas 1.80 to 3.80 for low pollen hoarding colonies (Table 4). The main criteria for selecting colonies with high pollen hoarding tendencies and low pollen hoarding tendencies were average pollen stores. The three high pollen hoarding colonies (H-4, H-5 and H-9) were those which scored 3 to 4.50 whereas low pollen hoarding colonies (L-4, L-6 and L9) scored 4 to 4.50 for average pollen stores and bee foragers. The selected colonies were gentle, disease free and maintained cleanliness during the period under observations.

Variations in colony parameters like colony strength, brood area, pollen stores, honey stores in A. mellifera have already been discussed. Hygienic behaviour for selecting Varroa tolerant colonies and pollen hoarding and pollen foraging preference have been considered as basis for selection of colonies (Rinderer, 1986). The present finding on pin killed brood got support from the findings of 
Sharma (2010), who found that hygienic behaviour varied from 48 to $96 \%$ (February March), 65.33 to 97.33 per cent (April - June) and 42.67 to 97.33 per cent (July-September). Kumar and Mall (2017) have also reported that percentage of pin killed brood removed after 24 hours ranged from 35.33 to 39.85 per cent.

Hellmich et al., (1985) have also made selection for high and low pollen hoarding honeybees and selected three sublines of high pollen hoarders and 3 with low pollen hoarders. They have reported differences in amount of pollen hoarded in different strains of honeybees.
Singh et al., (2007) have also selected better performing colonies on the basis of scores and their findings revealed that three out of ten colonies selected from PAU, Ludhiana campus apiary,L3 colony obtained highest overall mean score (3.720).

Except temperament (4.875) and hoarding efficiency (2.750), the score for other parameters such as cleanliness (5.00), honey stores (3.175), pollen foragers / 5 min (2.50) and pollen stores (3.125) were the highest for this colony (L3).

The scoring for temperament and hoarding efficiency were also reasonably good.

Table.1 Performance of high pollen hoarding colonies during August, 2016 to February, 2017

\begin{tabular}{|c|c|c|c|c|c|c|}
\hline \multirow[b]{2}{*}{$\begin{array}{c}\text { Colony } \\
\text { No. }\end{array}$} & \multicolumn{5}{|c|}{ Colony parameters at 21 days interval } & \multirow[b]{2}{*}{$\begin{array}{c}\text { Average } \\
\text { pollen } \\
\text { stores at } \\
10 \text { days } \\
\text { interval } \\
\left(\mathrm{cm}^{2}\right)\end{array}$} \\
\hline & $\begin{array}{c}\text { Average } \\
\text { colony strength } \\
\text { (No. of frames } \\
\text { with bees) }\end{array}$ & $\begin{array}{c}\text { Average brood } \\
\text { area } \\
\left(\mathrm{cm}^{2}\right)\end{array}$ & $\begin{array}{c}\begin{array}{c}\text { Average pollen } \\
\text { stores } \\
\left(\mathrm{cm}^{2}\right)\end{array}\end{array}$ & $\begin{array}{l}\text { Average honey } \\
\text { stores } \\
\text { (g) }\end{array}$ & $\begin{array}{c}\text { Average } \\
\text { hygienic } \\
\text { behaviour } \\
(\%)\end{array}$ & \\
\hline $\mathrm{H} 1$ & 7.86 & $\begin{array}{c}2723.43 \\
(3.40)^{*}\end{array}$ & $\begin{array}{c}281.36^{* * *} \\
(2.28)\end{array}$ & $\begin{array}{c}758.00 \\
(2.78)\end{array}$ & $\begin{array}{c}89.14 \\
(78.09)^{* *}\end{array}$ & $\begin{array}{c}317.79^{* * * * *} \\
(2.32)\end{array}$ \\
\hline $\mathrm{H} 2$ & 4.43 & $\begin{array}{c}1074.29 \\
(2.62) \\
\end{array}$ & $\begin{array}{c}347.00 \\
(2.39) \\
\end{array}$ & $\begin{array}{l}513.57 \\
(2.64) \\
\end{array}$ & $\begin{array}{c}92.29 \\
(79.46) \\
\end{array}$ & $\begin{array}{c}362.78 \\
(2.41) \\
\end{array}$ \\
\hline $\mathrm{H} 3$ & 5.57 & $\begin{array}{c}2275.21 \\
(3.34) \\
\end{array}$ & $\begin{array}{r}204.61 \\
(2.07) \\
\end{array}$ & $\begin{array}{c}197.43 \\
(1.67) \\
\end{array}$ & $\begin{array}{c}89.71 \\
(77.82) \\
\end{array}$ & $\begin{array}{l}377.02 \\
(2.27) \\
\end{array}$ \\
\hline $\mathrm{H} 4$ & 6.93 & $\begin{array}{c}2930.93 \\
(3.44) \\
\end{array}$ & $\begin{array}{l}485.61 \\
(2.26) \\
\end{array}$ & $\begin{array}{l}514.29 \\
(2.62) \\
\end{array}$ & $\begin{array}{c}96.00 \\
(83.74) \\
\end{array}$ & $\begin{array}{l}484.71 \\
(2.41) \\
\end{array}$ \\
\hline H5 & 8.29 & $\begin{array}{c}2585.79 \\
(3.37) \\
\end{array}$ & $\begin{array}{l}613.36 \\
(2.62)\end{array}$ & $\begin{array}{c}1045.00 \\
(2.92)\end{array}$ & $\begin{array}{c}69.57 \\
(58.63) \\
\end{array}$ & $\begin{array}{l}660.43 \\
(2.61)\end{array}$ \\
\hline H6 & 6.57 & $\begin{array}{c}2240.86 \\
(3.32) \\
\end{array}$ & $\begin{array}{l}370.21 \\
(2.39) \\
\end{array}$ & $\begin{array}{c}401.86 \\
(2.15) \\
\end{array}$ & $\begin{array}{c}91.14 \\
(81.59) \\
\end{array}$ & $\begin{array}{l}439.31 \\
(2.44) \\
\end{array}$ \\
\hline $\mathrm{H} 7$ & 6.14 & $\begin{array}{c}2654.79 \\
(3.39)\end{array}$ & $\begin{array}{l}352.36 \\
(2.44)\end{array}$ & $\begin{array}{c}633.00 \\
(2.68)\end{array}$ & $\begin{array}{c}83.71 \\
(72.06)\end{array}$ & $\begin{array}{l}372.81 \\
(2.45)\end{array}$ \\
\hline $\mathrm{H} 8$ & 6.00 & $\begin{array}{c}2712.64 \\
(3.39) \\
\end{array}$ & $\begin{array}{c}134.36 \\
(1.99) \\
\end{array}$ & $\begin{array}{c}534.86 \\
(2.60) \\
\end{array}$ & $\begin{array}{c}87.14 \\
(74.61) \\
\end{array}$ & $\begin{array}{l}184.05 \\
(2.10) \\
\end{array}$ \\
\hline H9 & 7.43 & $\begin{array}{c}3052.71 \\
(3.45) \\
\end{array}$ & $\begin{array}{l}628.29 \\
(2.48) \\
\end{array}$ & $\begin{array}{l}686.43 \\
(2.77) \\
\end{array}$ & $\begin{array}{c}88.57 \\
(75.04) \\
\end{array}$ & $\begin{array}{l}450.48 \\
(2.41) \\
\end{array}$ \\
\hline $\mathrm{H} 10$ & 6.00 & $\begin{array}{c}2522.93 \\
(3.35) \\
\end{array}$ & $\begin{array}{c}324.04 \\
(2.3) \\
\end{array}$ & $\begin{array}{r}257.29 \\
(1.98) \\
\end{array}$ & $\begin{array}{c}92.86 \\
(79.81) \\
\end{array}$ & $\begin{array}{l}380.31 \\
(2.32) \\
\end{array}$ \\
\hline $\mathbf{C D}_{(0.05)}$ & 1.09 & 0.44 & NS & 0.64 & NS & NS \\
\hline
\end{tabular}

*Figures in parentheses are $\log (\mathrm{x}+1)$ transformed values; **Figures in parentheses are arc sin transformed values 
Table.2 Performance of low pollen hoarding colonies during August, 2016 to February, 2017

\begin{tabular}{|c|c|c|c|c|c|c|}
\hline \multirow[b]{2}{*}{ Colony No. } & \multicolumn{5}{|c|}{ Colony parameters at 21 days interval } & \multirow[b]{2}{*}{$\begin{array}{l}\text { Average pollen } \\
\text { stores**** } \\
\left(\mathbf{c m}^{2}\right)\end{array}$} \\
\hline & $\begin{array}{c}\text { Average colony } \\
\text { strength } \\
\text { (No. of frames } \\
\text { with bees) }\end{array}$ & $\begin{array}{c}\text { Average brood } \\
\text { area } \\
\left(\mathrm{cm}^{2}\right)\end{array}$ & $\begin{array}{c}\text { Average } \\
\text { pollen } \\
\text { stores } * * * \\
\left(\mathrm{~cm}^{2}\right)\end{array}$ & $\begin{array}{l}\text { Average } \\
\text { honey } \\
\text { stores } \\
\text { (g) }\end{array}$ & $\begin{array}{c}\text { Average } \\
\text { hygienic } \\
\text { behaviour } \\
(\%)\end{array}$ & \\
\hline L1 & 4.71 & $\begin{array}{l}1204.07 \\
(2.85)^{*}\end{array}$ & $\begin{array}{c}366.00 \\
(1.99) \\
\end{array}$ & $\begin{array}{r}134.57 \\
(1.51) \\
\end{array}$ & $\begin{array}{c}83.14 \\
(70.48)^{* *}\end{array}$ & $\begin{array}{c}350.10 \\
(2.03) \\
\end{array}$ \\
\hline L2 & 5.36 & $\begin{array}{c}1777.21 \\
(3.18)\end{array}$ & $\begin{array}{c}357.64 \\
(2.13)\end{array}$ & $\begin{array}{l}318.00 \\
(2.15)\end{array}$ & $\begin{array}{c}79.14 \\
(67.21)\end{array}$ & $\begin{array}{c}382.88 \\
(2.09)\end{array}$ \\
\hline L3 & 4.71 & $\begin{array}{c}1749.64 \\
(3.18) \\
\end{array}$ & $\begin{array}{c}214.86 \\
(1.93)\end{array}$ & $\begin{array}{c}305.43 \\
(2.31) \\
\end{array}$ & $\begin{array}{c}86.86 \\
(71.22) \\
\end{array}$ & $\begin{array}{r}435.90 \\
(2.28) \\
\end{array}$ \\
\hline L4 & 4.43 & $\begin{array}{c}1160.71 \\
(2.60) \\
\end{array}$ & $\begin{array}{r}389.07 \\
(2.29) \\
\end{array}$ & $\begin{array}{c}397.29 \\
(2.14) \\
\end{array}$ & $\begin{array}{c}79.71 \\
(67.68) \\
\end{array}$ & $\begin{array}{l}260.02 \\
(2.14) \\
\end{array}$ \\
\hline L5 & 5.79 & $\begin{array}{c}2184.36 \\
(3.24)\end{array}$ & $\begin{array}{c}379.50 \\
(2.23)\end{array}$ & $\begin{array}{l}322.71 \\
(2.09)\end{array}$ & $\begin{array}{c}80.00 \\
(69.79) \\
\end{array}$ & $\begin{array}{c}370.83 \\
(2.18)\end{array}$ \\
\hline L6 & 5.21 & $\begin{array}{c}1913.36 \\
(2.81) \\
\end{array}$ & $\begin{array}{c}233.14 \\
(1.71) \\
\end{array}$ & $\begin{array}{c}255.43 \\
(1.76) \\
\end{array}$ & $\begin{array}{c}71.57 \\
(63.20) \\
\end{array}$ & $\begin{array}{c}238.33 \\
(1.77) \\
\end{array}$ \\
\hline L7 & 6.21 & $\begin{array}{c}2627.00 \\
(3.36) \\
\end{array}$ & $\begin{array}{l}531.68 \\
(2.08) \\
\end{array}$ & $\begin{array}{l}270.57 \\
(1.76) \\
\end{array}$ & $\begin{array}{c}78.86 \\
(68.99) \\
\end{array}$ & $\begin{array}{l}523.81 \\
(1.99) \\
\end{array}$ \\
\hline L8 & 4.29 & $\begin{array}{c}1369.57 \\
(2.58) \\
\end{array}$ & $\begin{array}{c}217.46 \\
(1.64) \\
\end{array}$ & $\begin{array}{l}76.14 \\
(1.18) \\
\end{array}$ & $\begin{array}{c}67.86 \\
(58.81) \\
\end{array}$ & $\begin{array}{c}300.74 \\
(1.71) \\
\end{array}$ \\
\hline L9 & 6.29 & $\begin{array}{c}2083.14 \\
(2.46) \\
\end{array}$ & $\begin{array}{c}343.36 \\
(1.93) \\
\end{array}$ & $\begin{array}{r}271.57 \\
(1.99) \\
\end{array}$ & $\begin{array}{c}71.14 \\
(64.28) \\
\end{array}$ & $\begin{array}{c}250.41 \\
(1.56) \\
\end{array}$ \\
\hline L10 & 5.00 & $\begin{array}{c}2012.00 \\
(3.23) \\
\end{array}$ & $\begin{array}{c}446.36 \\
(2.51)\end{array}$ & $\begin{array}{c}252.86 \\
(2.34)\end{array}$ & $\begin{array}{c}93.14 \\
(79.55) \\
\end{array}$ & $\begin{array}{l}477.48 \\
(2.40) \\
\end{array}$ \\
\hline $\mathrm{CD}_{(0.05)}$ & 1.24 & NS & 0.43 & 0.70 & $\mathrm{NS}$ & 0.40 \\
\hline
\end{tabular}

*Figures in parentheses are $\log (\mathrm{x}+1)$ transformed values; **Figures in parentheses are arc sin transformed values

Table.3 Ranking of 20 A. melifera colonies on 5 point scale for various parameters

\begin{tabular}{|c|c|c|c|c|c|c|}
\hline \multirow[b]{2}{*}{ Colony No. } & \multicolumn{5}{|c|}{ Scored value on 5 point scale } & \multirow[b]{2}{*}{$\begin{array}{c}\text { Mean } \\
(\mathbf{S}, \mathbf{B}, \mathbf{P}, \mathbf{H}, \mathbf{H B})\end{array}$} \\
\hline & $\begin{array}{l}\text { Average } \\
\text { colony } \\
\text { strength } \\
\text { (S) }\end{array}$ & $\begin{array}{c}\text { Average } \\
\text { brood area } \\
\text { (B) }\end{array}$ & $\begin{array}{l}\text { Average } \\
\text { pollen } \\
\text { stores* } \\
\text { (P) }\end{array}$ & $\begin{array}{c}\text { Average } \\
\text { honey stores } \\
\text { (H) }\end{array}$ & $\begin{array}{c}\text { Average } \\
\text { hygienic } \\
\text { behavior } \\
\text { (HB) }\end{array}$ & \\
\hline H1 & 5 & 5 & 2 & 4 & 4 & 4.00 \\
\hline H2 & 1 & 1 & 3 & 2 & 5 & 2.40 \\
\hline H3 & 2 & 4 & 1 & 1 & 4 & 2.40 \\
\hline H4 & 4 & 5 & 4 & 2 & 5 & 4.00 \\
\hline H5 & 5 & 4 & 5 & 5 & 1 & 4.00 \\
\hline H6 & 3 & 3 & 3 & 2 & 5 & 3.20 \\
\hline H7 & 3 & 4 & 3 & 3 & 3 & 3.20 \\
\hline H8 & 3 & 5 & 1 & 3 & 4 & 3.20 \\
\hline H9 & 4 & 5 & 5 & 3 & 4 & 4.20 \\
\hline H10 & 3 & 4 & 3 & 1 & 5 & 3.20 \\
\hline L1 & 2 & 1 & 3 & 1 & 4 & 2.20 \\
\hline L2 & 4 & 3 & 3 & 4 & 3 & 3.40 \\
\hline L3 & 3 & 3 & 5 & 4 & 4 & 3.80 \\
\hline L4 & 2 & 1 & 3 & 5 & 3 & 2.80 \\
\hline L5 & 4 & 4 & 3 & 4 & 3 & 3.60 \\
\hline L6 & 2 & 3 & 5 & 3 & 1 & 2.80 \\
\hline L7 & 5 & 5 & 1 & 4 & 3 & 3.60 \\
\hline L8 & 1 & 1 & 5 & 1 & 1 & 1.80 \\
\hline L9 & 5 & 4 & 3 & 4 & 1 & 3.40 \\
\hline L10 & 4 & 3 & 2 & 3 & 5 & 3.40 \\
\hline
\end{tabular}


Table.4 Ranking of 20 A. melifera colonies on 5 point scale for various parameters

\begin{tabular}{|c|c|c|c|}
\hline \multirow{2}{*}{ Colony No. } & \multicolumn{2}{|c|}{ Scored value on 5 point scale } & \multirow{2}{*}{ Mean } \\
\cline { 2 - 3 } & Average pollen stores & Average incoming pollen foragers & \\
\hline H1 & 2 & 4 & 3.00 \\
\hline H2 & 2 & 1 & 1.50 \\
\hline H3 & 3 & 2 & 2.50 \\
\hline H5 & 4 & 2 & 3.00 \\
\hline H6 & 5 & 4 & 4.50 \\
\hline H7 & 3 & 2 & 2.50 \\
\hline H9 & 2 & 2 & 2.00 \\
\hline H10 & 1 & 3 & 2.00 \\
\hline L1 & 3 & 5 & 4.00 \\
\hline L2 & 3 & 1 & 2.00 \\
\hline L4 & 4 & 5 & 4.50 \\
\hline L5 & 3 & 3 & 3.00 \\
\hline L6 & 2 & 3 & 2.50 \\
\hline L7 & 5 & 4 & 4.50 \\
\hline L8 & 3 & 2 & 2.50 \\
\hline L9 & 5 & 3 & 4.00 \\
\hline L10 & 1 & 1 & 1.00 \\
\hline
\end{tabular}

Table.5 Bee activity of high and low pollen hoarding colonies from August, 2016 to February, 2017

\begin{tabular}{|c|c|c|c|c|c|c|}
\hline \multirow{3}{*}{ Colony No. } & \multicolumn{6}{|c|}{ Number of incoming bees (with pollen load) and outgoing bees/ minute at hive entrance } \\
\hline & \multicolumn{2}{|c|}{ Incoming } & \multirow{2}{*}{ Mean } & \multicolumn{2}{|c|}{ Outgoing } & \multirow{2}{*}{ Mean } \\
\hline & HPH* & LPH** & & HPH & LPH & \\
\hline H1/L1 & 57.66 & 36.14 & 46.90 & 71.14 & 40.85 & 56.00 \\
\hline H2/L2 & 45.71 & 45.57 & 45.64 & 64.57 & 52.85 & 58.71 \\
\hline H3/L3 & 49.43 & 42.04 & 45.73 & 52.47 & 50.38 & 51.43 \\
\hline H4/L4 & 51.23 & 40.76 & 46.00 & 65.47 & 46.57 & 56.02 \\
\hline H5/L5 & 56.09 & 48.05 & 52.07 & 62.28 & 54.90 & 58.59 \\
\hline H6/L6 & 50.33 & 43.62 & 46.97 & 56.19 & 48.85 & 52.52 \\
\hline H7/L7 & 49.14 & 53.62 & 51.38 & 59.62 & 63.00 & 61.31 \\
\hline H8/L8 & 51.85 & 40.28 & 46.07 & 61.24 & 44.95 & 53.09 \\
\hline H9/L9 & 60.90 & 44.05 & 52.47 & 77.04 & 51.52 & 64.28 \\
\hline H10/L10 & 47.09 & 34.09 & 40.59 & 50.62 & 40.00 & 45.31 \\
\hline Mean & 51.94 & 42.82 & & 62.06 & 49.39 & \\
\hline $\mathbf{C D} D_{(0.05)}$ & \multicolumn{3}{|c|}{$\begin{array}{l}\text { Pollen hoarding }=4.24 \text {, Colony }=\text { NS, Activity } \\
\text { x Colony = NS; }\end{array}$} & \multicolumn{3}{|c|}{$\begin{array}{l}\text { Pollen hoarding }=5.40, \text { Colony }=\text { NS }, \text { Activit } \\
\text { x Colony }=\text { NS }\end{array}$} \\
\hline
\end{tabular}

*High pollen hoarding; ** Low pollen hoarding 
Table.6 Performance of selected colonies from general stocks of A. mellifera in apple orchard at Mashobra, Shimla during April, 2017

\begin{tabular}{|c|c|c|c|c|}
\hline \multirow[b]{2}{*}{ Colony No. } & \multicolumn{4}{|c|}{ Colony parameters } \\
\hline & $\begin{array}{l}\text { Average colony } \\
\text { strength } \\
\text { (No. of bees } \\
\text { frames) }\end{array}$ & $\begin{array}{c}\text { Average } \\
\text { brood Area } \\
\left(\mathbf{c m}^{2}\right)\end{array}$ & $\begin{array}{c}\text { Average } \\
\text { pollen store } \\
\left(\mathbf{c m}^{2}\right)\end{array}$ & $\begin{array}{c}\text { Average } \\
\text { honey Store } \\
\text { (g) }\end{array}$ \\
\hline $\mathbf{H} 4 *$ & 7.00 & $\begin{array}{c}1821.25 \\
(3.26) * * * *\end{array}$ & 47.00 & $\begin{array}{c}550.00 \\
(2.69)\end{array}$ \\
\hline H5 & 7.00 & $\begin{array}{c}1510.00 \\
(3.17)\end{array}$ & 62.50 & $\begin{array}{c}300.00 \\
(2.35)\end{array}$ \\
\hline H9 & 7.50 & $\begin{array}{c}1970.00 \\
(3.29)\end{array}$ & 46.00 & $\begin{array}{l}500.00 \\
(2.48)\end{array}$ \\
\hline $\mathbf{L} 4 * *$ & 4.50 & $\begin{array}{l}778.75 \\
(2.89)\end{array}$ & 27.00 & $\begin{array}{c}550.00 \\
(2.74)\end{array}$ \\
\hline L6 & 6.00 & $\begin{array}{c}2140.00 \\
(3.33)\end{array}$ & 44.00 & $\begin{array}{c}475.00 \\
(2.65)\end{array}$ \\
\hline L9 & 6.00 & $\begin{array}{l}613.75 \\
(2.13)\end{array}$ & 76.00 & $\begin{array}{l}550.00 \\
(2.69)\end{array}$ \\
\hline G1*** & 7.50 & $\begin{array}{c}1930.00 \\
(3.29)\end{array}$ & 29.00 & $\begin{array}{c}350.00 \\
(2.39) \\
\end{array}$ \\
\hline G2 & 5.00 & $\begin{array}{l}823.75 \\
(2.80)\end{array}$ & 23.00 & $\begin{array}{c}250.00 \\
(1.35)\end{array}$ \\
\hline G3 & 6.50 & $\begin{array}{c}1110.00 \\
(3.05)\end{array}$ & 27.18 & $\begin{array}{c}275.00 \\
(2.44)\end{array}$ \\
\hline $\mathrm{CD}_{(0.05)}$ & 0.57 & 0.57 & 23.18 & 0.69 \\
\hline
\end{tabular}

* High pollen hoarding; ** Low pollen hoarding; *** General pollen hoarding $* * * *$ Figures in parentheses are $\log (\mathrm{x}+1)$ transformed values

Table.7 Percentage of incoming bees with pollen in apple orchard at Mashobra, Shimla during April, 2017

\begin{tabular}{|c|c|}
\hline Colony No. & No. of bees with pollen / minute \\
\hline H4* & 23.11 \\
\hline H5 & 17.32 \\
\hline H9 & 17.67 \\
\hline L4** & 19.20 \\
\hline L6 & 19.59 \\
\hline L9 & 13.15 \\
\hline G1*** & 23.26 \\
\hline G2 & 9.95 \\
\hline G3 & 15.99 \\
\hline CD $(\mathbf{0 . 0 5 )}$ & $\mathbf{5 . 1 0}$ \\
\hline
\end{tabular}

* High pollen hoarding; ** Low pollen hoarding; *** General pollen hoarding 
Table.8 Percentage of incoming bees with apple pollen during onset, full and end of bloom in apple orchard at Mashobra, Shimla during April, 2017

\begin{tabular}{|c|c|c|c|c|}
\hline \multirow{2}{*}{ Colony No. } & \multicolumn{3}{|c|}{ Percentage of incoming bees with apple pollen } & \multirow{2}{*}{ Mean } \\
\hline & Onset of bloom & Full bloom & End of bloom & \\
\hline $\mathrm{H} 4 *$ & $\begin{array}{c}10.67 \\
(3.33)^{*} \\
\end{array}$ & $\begin{array}{l}19.00 \\
(4.19) \\
\end{array}$ & $\begin{array}{c}0.67 \\
(1.24)\end{array}$ & $\begin{array}{l}10.11 \\
(2.92)\end{array}$ \\
\hline H5 & $\begin{array}{l}17.33 \\
(4.26) \\
\end{array}$ & $\begin{array}{l}22.00 \\
(4.80) \\
\end{array}$ & $\begin{array}{c}0.67 \\
(1.24) \\
\end{array}$ & $\begin{array}{l}13.33 \\
(3.44) \\
\end{array}$ \\
\hline H9 & $\begin{array}{l}12.67 \\
(3.61) \\
\end{array}$ & $\begin{array}{l}22.00 \\
(4.78)\end{array}$ & $\begin{array}{c}3.33 \\
(1.96)\end{array}$ & $\begin{array}{l}12.67 \\
(3.45)\end{array}$ \\
\hline $\mathrm{L} 4 * *$ & $\begin{array}{l}14.67 \\
(3.79)\end{array}$ & $\begin{array}{l}17.00 \\
(4.19)\end{array}$ & $\begin{array}{c}4.00 \\
(2.21)\end{array}$ & $\begin{array}{l}11.89 \\
(3.39)\end{array}$ \\
\hline L6 & $\begin{array}{l}18.67 \\
(4.32) \\
\end{array}$ & $\begin{array}{l}12.00 \\
(3.58) \\
\end{array}$ & $\begin{array}{c}5.33 \\
(2.49) \\
\end{array}$ & $\begin{array}{l}12.00 \\
(3.46) \\
\end{array}$ \\
\hline L9 & $\begin{array}{l}16.67 \\
(4.16) \\
\end{array}$ & $\begin{array}{c}9.00 \\
(3.09) \\
\end{array}$ & $\begin{array}{l}4.00 \\
(2.21) \\
\end{array}$ & $\begin{array}{r}9.89 \\
(3.15) \\
\end{array}$ \\
\hline $\mathrm{G} 1 * * *$ & $\begin{array}{l}15.33 \\
(3.94) \\
\end{array}$ & $\begin{array}{l}20.00 \\
(4.58)\end{array}$ & $\begin{array}{c}3.33 \\
(1.77) \\
\end{array}$ & $\begin{array}{l}12.89 \\
(3.43) \\
\end{array}$ \\
\hline $\mathrm{G} 2$ & $\begin{array}{l}22.67 \\
(4.86)\end{array}$ & $\begin{array}{l}27.00 \\
(5.19)\end{array}$ & $\begin{array}{c}6.67 \\
(2.73)\end{array}$ & $\begin{array}{l}18.78 \\
(4.26)\end{array}$ \\
\hline G3 & $\begin{array}{l}17.33 \\
(4.24) \\
\end{array}$ & $\begin{array}{l}21.00 \\
(4.49) \\
\end{array}$ & $\begin{array}{c}2.00 \\
(1.66) \\
\end{array}$ & $\begin{array}{l}13.44 \\
(3.46) \\
\end{array}$ \\
\hline Mean & $\begin{array}{l}16.22 \\
(4.06) \\
\end{array}$ & $\begin{array}{l}18.78 \\
(4.32) \\
\end{array}$ & $\begin{array}{c}3.33 \\
(1.95) \\
\end{array}$ & \\
\hline
\end{tabular}

$\mathrm{CD}_{(\mathbf{0 . 0 5})}$ Blooming period (0.28); Colony (0.48); Blooming period x Colony (0.83)

* High pollen hoarding; ** Low pollen hoarding;*** General pollen hoarding; **** Figures in parentheses are square root transformed values

\section{Bee activity at hive entrance}

Number of incoming bees with pollen loads (Table 5) was significantly more (51.94 bees per minute) in high pollen hoarding honeybee colonies as compared to low pollen hoarding honeybee colonies ( 42.82 bees per minute). Similarly the number of outgoing bees was statistically higher in high pollen hoarding honeybees (62.06 bees per minute) as compared to low pollen hoarding honeybees (49.39 bees per minute).

Chaudhary and Gupta (2000) have reported significant difference in incoming and outgoing foragers at hive entrance in $A$. mellifera colonies. They found that average number of incoming bee was significantly more in high brood survival as compared to medium brood survival and low brood survival colonies. Nagaraja (2000) have also studied the annual foraging cycle of $A$. mellifera and reported that foraging activity of A. mellifera showed fluctuations within colonies and seasons.

\section{Evaluation of selected $A$. mellifera for apple foraging preference}

Average colony strength was statistically same (7.50 bee frames) in colony number G-1 and H-9. The minimum colony strength was recorded in L-4 (4.50 bee frames) and G-2 (5.00 bee frames). The average maximum brood area was recorded in colony number L$6\left(2140 \mathrm{~cm}^{2}\right)$. The colony number assigned as L-9 reared significantly less brood (613.75 $\mathrm{cm}^{2}$ ). The average maximum amount of pollen stores were recorded in colony number L-9 $\left(76.00 \mathrm{~cm}^{2}\right)$ and $\mathrm{H}-5\left(62.50 \mathrm{~cm}^{2}\right)$. The minimum amount of pollen was stored in colony number G-2 $\left(23 \mathrm{~cm}^{2}\right)$. Maximum 
amount of honey was stored in colony number L-4 whereas, the minimum was stored in colony number G-2 (250 g).

Hellmich et al., (1985) have also selected colonies for alfalfa foraging preference. They found that high and low lines selected for pollen foraging for alfalfa were significantly different. High pollen hoarders averaged $287.10 \mathrm{~cm}^{2}$ of pollen while low pollen hoarders averaged $65.80 \mathrm{~cm}^{2}$.

The variations in colony parameters of selected colonies are also in agreement with earlier observation on the performance of six selected A. mellifera colonies at Nauni, Solan (Anonymous, 2013). These observations revealed variations in colony strength (7.30 to 8.90 bee frames), brood area (929.47 to $1282.40 \mathrm{~cm}^{2}$ ), pollen area (122.17 to 199.51 $\mathrm{cm}^{2}$ ) and honey stores (838.88 to $3297.77 \mathrm{~g}$ ).

\section{Pollen foraging in selected $A$. mellifera colonies}

The percentage of incoming bees with pollen (Table 7) during flowering period in apple orchard at Mashobra, Shimla during April, 2017 revealed that colony number G-1 had higher percentage of incoming bees with pollen / minute $(23.26 \%)$ followed by $\mathrm{H}-4$ (23.11\%), L-6(19.59\%) and L-4 (19.20\%). The average minimum number of incoming bees with pollen per minute was recorded in colony number G-2 (9.95\%) and L-9 (13.15 $\%)$.

These observations are partly in agreement with Chaudhary et al., (2000), who have reported significant difference in incoming foragers at hive entrance in A. mellifera colonies. The average number of incoming bee was significantly more in high brood survival as compared to medium brood survival and low brood survival colonies indicating that the incoming foragers varied with colony conditions specially with respect to brood area and pollen stores.

Studies on apple pollen preference in $A$. mellifera

The data on apple pollen preference revealed that colony number G-2 showed highest preference for apple pollen foraging with 18.78 per cent of apple pollen loads. Colony $\mathrm{H}-4$ recorded minimum percentage of apple pollen load. Out of nine, seven colonies collected significantly same percentage of apple pollen. These honeybee were from colony number G-3, L-6, H-9, H-5, G-1, L-4 and L-9 with 13.44, 12.00, 12.67, 13.33, $12.89,11.89$ and 9.89 per cent apple pollen.

Mackensen and Nye (1966) were the first to select honeybees for pollen preferences. Two - way selection produced colonies with high percentage of alfalfa pollen collecting bees. Similarly, Hellmich et al., (1985) found that high and low lines selected for pollen foraging for alfalfa were significantly different. The present findings on variability in A. mellifera for apple pollen foraging got support from the earlier observations on preference of $A$. mellifera colonies for apple pollen (Annonymous, 2015). These observations recorded at four different locations of Kullu valley revealed that the preference of A. mellifera colonies for apple pollen varied from 4.30 to 97.62 per cent in general stock of $A$. mellifera. The present studies further revealed that colonies with high pollen hoarding had significant higher number of incoming pollen bees but the same was not true for preference for apple pollen foraging for A. mellifera. High performance of G-2 from general stock might be due to the fact that the colonies with high pollen hoarding may not have preference for apple pollen and they foraged on available competing flora. These findings got support from earlier observations of Dag et al., (2005) 
and support their hypothesis regarding the possibility of genetic component to honeybee preference for apple pollen (Tables 6-8).

Similar to present findings, the preference for collecting pollen from the target crop was found for alfalfa (Nye and Makensen, 1970) and sunflower (Basualdo et al., 2000). Thus selection efforts for bees with the preference for apple pollen could be combined with selection for pollen hoarding to get a strain of honeybee with higher preference for apple pollen foraging.

The data recorded on twenty selected colonies suggested that $A$. mellifera colonies varied in their pollen hoarding tendencies which shows that pollen hoarding by honeybee can be modified by selection. A large proportion of honeybees from experimental colonies placed in apple orchard at Mashobra, collected pollen from competing flora. However, the experimental colonies showed varied preference for apple pollen which indicates that there is possibility of selecting $A$. mellifera with high apple pollen foraging preference.

\section{References}

Ambrose, J.T., 1990. Apple pollination. In: NC Apple Production Mannual. North Carolina Agriculture Extension Service, AG, 415pp.

Anonymous, 2013. Annual Report: All India Coordinated project on honey bees and pollinators. $31 \mathrm{p}$.

Anonymous, 2015. Horticulture development in Himachal Pradesh at a glance. www.hpagrisnet.gov.in

Basualdo, M., Arrasbure BE and Jong DE. 2000. Africanized honey bees (Hymenoptera: Apidae) have a greater fidelity to sunflowers than European bees. Journal of Economic Entomology 93:304-307.
Brittain, W.H., 1933. Apple pollination studies in the Annapolis Valley, Nova Scotia. Canadian Department of Agriculture Bulletin, New Series 162:1198.

Chaudhary, S., and Gupta JK. 2000. Influence of worker brood survival on the morphoiogical, behavioural and economical characters of Apis mellifera. Indian Bee Journal. 62:55-58.

Dag, A., Stern AR and Shafir S. 2005. Honey bee (Apis mellifera) strains differ in apple (Malus domestica) pollen foraging preference. Journal of Apicultural Research 44:15-20.

Gupta, J.K., Goyal NP, Sharma JP and Gautam DR. 1993. The effect of placement of varying numbers of Apis mellifera colonies on the fruit set in apple orchards having different proportions of pollinisers. In: (Veeresh GK, Shankar RU and Ganeshaiah KN eds). Proceedings of the International Symposium on Pollination in the Tropics pp.197-201.

Hellmich, R.L., Kulincevic JM and Rothenbuhler WC. 1985. Selection for high and low pollen hoarding honey bees. The Journal of Heredity 76:155158.

Kendall, D.A., 1973. Viability and compatibility of pollen on insects visiting apple blossom. Journal of Applied Ecology 10:847-853.

Kumar, J., and Mall P. 2017. Hygienic behavioral response of Apis mellifera $\mathrm{L}$. against pin-killed and infectious brood. Journal of Entomology and Zoology Studies 5:913-915.

Mackensen, O., and Nye WP. 1966. Selecting and breeding honeybees for collecting alfalfa pollen. Journal of Apicultural Research 5:79-86.

Nagaraja, N., 2000. Studies on annual foraging cycle of Apis mellifera L. Indian Bee Journal 62:11-17. 
Page, R.A., Fondrk MK, Hunt GT, Guzman NE, Humphries MA, Nguyen $K$ and Greene AS. 2000. Genetic dissection of honey bees (Apis mellifera L.) foraging behavior. Journal of Heredity 91:474479.

Rana, V.K., and Kumar S. 2011. Performance of Apis mellifera L. colonies in the high hills of Himachal Pradesh. Indian Journal of Entomology 73:241-243.

Rinderer, T.E., 1986. Selection In: Bee genetics and Breeding. (T E Rinderer Ed.). Academic press, INC, (London) Ltd, Orlando, Florida. 409p.

Robinson, W.S., 1979. Effect of apple cultivar on foraging behavior and pollen transfer by honey bees. Journal of the American Society for Horticultural Science 104:596-598.

Sharma, R., 2010. Screening of Apis mellifera L. colonies for Varroa tolerance and evalustion of colony perforance of selected stock. Ph.D. Thesis. Dr YS Parmar University of Horticulture and Forestry, Nauni, Solan. 145p.

Singh, S., Gatoria GS and Chhuneja PK. 2007. Selection of best performing Apis Mellifera colonies for stock improvement programme. Indian Bee Journal 69:1-7.

\section{How to cite this article:}

Harish Kumar Sharma, Monika, Kiran Rana and Meena Thakur. 2017. Selected High and Low Pollen Hoarding Colonies of Apis mellifera for Apple Foraging Preference. Int.J.Curr.Microbiol.App.Sci. 6(10): 2233-2244. doi: https://doi.org/10.20546/ijcmas.2017.610.264 